\title{
Human Socio-cultural Behavior Modeling: A Soft-Computing Approach
}

\author{
Tareq Z. Ahram ${ }^{1 *}$, Waldemar Karwowski ${ }^{2}$ \\ ,2 Institute for Advanced Systems Engineering (IASE) Department of Industrial Engineering and Management Systems University \\ of Central Florida, Orlando, Florida 32816, USA
}

\section{BIOGRAPHICAL NOTES}

Tareq Ahram, PhD, is the Research Manager and Lead Scientist working at the Institute for Advanced Systems Engineering (IASE), the Department of Industrial Engineering and Management Systems at the University of Central Florida. He holds Master of Science degree in Engineering Management (2004) from the University of Jordan, Master of Science degree in Human Engineering (2006) from the University of Central Florida, and Ph.D. (2008) in Industrial Engineering with specialization in Human Systems Integration and large-scale information retrieval systems (data analytics). Dr. Ahram research focuses primarily on human performance modeling, safety engineering and user experience research expert. Dr. Ahram received the 2012 Outstanding Researcher award from IBM. Dr. Ahram currently serves as the research manager and co-principal investigator projects funded by the Office of Naval Research, DoD and Industry in human performance modeling, human total ownership cost, human social modeling, complex nonlinear dynamic systems, intelligent systems and data analytics. Waldemar Karwowski, Ph.D., D.Sc., P.E., is Professor and Chairman, Department of Industrial Engineering and Management Systems and Executive Director of the Institute for Advanced Systems Engineering at the University of Central Florida, USA. He holds an M.S. (1978) in Production Engineering and Management from the Technical University of Wroclaw, Poland, and a Ph.D. (1982) in Industrial Engineering from Texas Tech University. He was awarded D.Sc. (dr habil.) degree in management science by the State Institute for Organization and Management in Industry, Poland (2004). He also received Honorary Doctorate degrees from three European universities. He is the author or coauthor of over 350 scientific publications, and is Certified Professional Ergonomist (BCPE). Dr. Karwowski was named the J. B. Speed School of Engineering Alumni Scholar for Research, University of Louisville (2004-2006). He served as member of the Board of Directors of the American Association of Engineering Societies (2006-2007). Dr. Karwowski currently serves on the Committee on Human Systems Integration, National Research Council of the National Academies, USA (2007-2009), and as Editor of the Human Factors and Ergonomics in Manufacturing journal, and Editor-in-Chief of Theoretical Issues in Ergonomics Science journal. He is Past President of the International Ergonomics Association (2000-2003), and Past President of the Human Factors and Ergonomics Society (2007).

\section{KEYWORDS}

Soft-Computing, Socio-cultural Modeling; Forecasting; Social Patterns; Human Social Culture Behavior; Cellular automata; Complex Systems.

\section{ABSTRACT}

Human behavioral and socio-cultural soft-computational modeling requires the abil- 
ity to represent and manipulate imprecise perceptual and cognitive concepts. The research investigation into soft-computational models of human behavior and socio-cultural interactions, account for natural vagueness of human thought and communication, along with the dynamics, complexity, uncertainty, variability and unpredictability of actions and behavior. This paper covers selected softcomputational methods which were investigated for the Human Social Culture Behavior (HSCB) modeling program with the emphasis on the powerful computational benefits and usefulness of these methods to support forecasting social patterns and building socio-cultural decision support systems. The paper also introduces a novel social networking analysis model for the next generation decision sup-port systems.

\section{Introduction}

Today's military focus is on the domain of irregular warfare and its companion security, stability, transition and reconstruction missions (Numrich and Tolk, 2010). According to Numrich and Tolk (2010) when irregular warfare tasks and missions are involved, avoiding the human, cultural and social elements invites failure. The need to include these elements has been documented and recognized in US military warfighting concepts. Human behavioral and socio-cultural models that deviate from a given norm can be interpreted as deviations when the behaviors or their consequences are not intentional and as violations when they are intentional. Such violations may act as warning indicators and signals that require further monitoring and deeper investigation (Schmorrow and Nicholson, 2010). According to the Irregular Warfare Joint Operating Concept IWJOC (2007): "Irregular warfare depends not just on our military prowess, but also our understanding of such social dynamics as tribal politics, social networks, religious influences and cultural mores." (IWJOC, 2007).

The military has made some adjustments to its force structure for recognizing the challenges based on irregular warfare. Bhattacharjee (2007) outlined a new Department of Defense (DoD) initiative for Human Social Culture Behavior (HSCB) modeling, designed to advanced current understanding of anthropological analyses and cultures interacted with during overseas operations (Drapeau and Mignone, 2007). The overarching goal of the HSCB Modeling is to support decision makers better understand and effectively operate in the human terrain during nonconventional warfare and other missions (HSCB, 2009). HSCB models are getting much attention in current and future operational requirements. Since HSCB models can be defined as a complex system, computational modeling and simulation techniques can deal with this complexity. Computational social scientists are researching how observations of human behavior might be used to develop scientifically based models of HSCB events (Schmorrow and Nicholson, 2011). HSCB models are designed to help explain and investigate the nonlinear and dynamic dependencies, structures, behavior, and associated trends along with organizational entities which can be considered as macro and micro level (Ahram and Karwowski, 2012a). According to Stanton (2007) and Ahram and Karwowski (2012a): Macro human social models address economies, socio-cultural regions, states, and political systems effects and micro models deal with ethnic tribes religious and military units at the tactical level.

\section{Soft-Computational Modeling}

Advances in computational socio-cultural network analysis (CSNA), social network analysis (SNA) and cultural domain analysis (CDA) will support the effective integration of soft computing, clustering and dimensionality reduction techniques. The overall research contribution for HSCB program is to develop and test a hybrid socio-cultural behavior assessment model and to design the software framework needed to enhance the ability of social and cultural researchers and military decision makers to interpret and explore mission's data so as to determine and predict anomalous individual and group behaviors, support extensive cultural situational awareness analysis for tactical decision-making and enable rapid socio-cultural assessment. Hunter et al. (2012) and Carrington and Scott (2011) confirmed the difficulty to pinpoint the genesis of the field of statistical and social network analysis. Researchers such as Goldenberg et al. (2009) and Kolaczyk (2009) provided a comprehensive examination and summary of research development in the field of social network analysis. However, the literature lacks the sufficient research and investigation of the benefits of applying softcomputational and mathematical techniques to 
human social network and cultural analysis.

Advances in computational social network analysis (CSNA), social network analysis (SNA) and cultural domain analysis (CDA) will support the effective integration of soft computing, clustering and dimensionality reduction techniques, such as, but not limited to, those techniques listed in this paper. This research paper covers HSCB research development of the socio-cultural behavior assessment model that directly benefits from recent advances in soft-computing and mathematical techniques, to aid in social network and cultural domain analyses, model imprecision and uncertainty in data, and determine anomalous behaviors (or activities) to support real-time computer based socio-cultural situational awareness and strategic decision making, rapid socio-cultural assessment, and training using visual and computational social network analysis methods in a far more efficient manner (Ahram and Karwowski, 2012b). The modeling approach presented in this paper is based on soft-computational social network analysis (CSNA) techniques and complex systems theory. The following selected soft-computational modeling techniques, Cellular Automata (CA), Evolving SelfOrganizing Maps and Artificial Neural Networks, were investigated for HSCB analysis and proofed beneficial as discussed in Section 3. This paper also identifies the software algorithms needed to enhance the ability of social and cultural researchers and military decision makers to interpret and explore mission specific cultural data so as to determine and predict anomalous individual and group behaviors, and support extensive cultural situational awareness analysis for tactical decisionmaking and rapid socio-cultural assessment.

\section{Forecasting with Socio-cultural Complex Net- work Analysis}

Complexity systems engineering is an emerging trans-disciplinary field of research that studies complex systems (Aslaksen, 2009; Cilliers, 1998). Complex systems have previously been studied in various fields including cybernetics, economics, sociology, anthropology, cognitive science, chemistry, mathematics, physics, systems science etc. Complexity itself is now the focus of much of the research in many fields - to explore what complexity is and what it means for understanding the systems that make up a social network.
Complexity provides a conceptual framework with which to view the world of socio-cultural network as demonstrated in Figure 1. Through it, research acknowledges that we are surrounded by things which are dynamic, changing, entangled, and interconnected. The study of complexity asks us to think in a holistic way, focusing on the connections between things rather than the things themselves. Complexity challenges us to move away from current intellectual habit of thinking linearly.

Complex systems are open, which means they depend on and contribute to their environment (Miller, \& Page 2007; Abraham et al. 2010, Knoke et al. 2008). In a complex system no one element has all the information. According to Knoke et al. (2008): "Each element in the system is ignorant of the behavior of the system as a whole, it responds only to the information that is available to it locally. Elements think locally, and act locally but their collective action produces the global intelligence of the system". This is known as distributed intelligence.

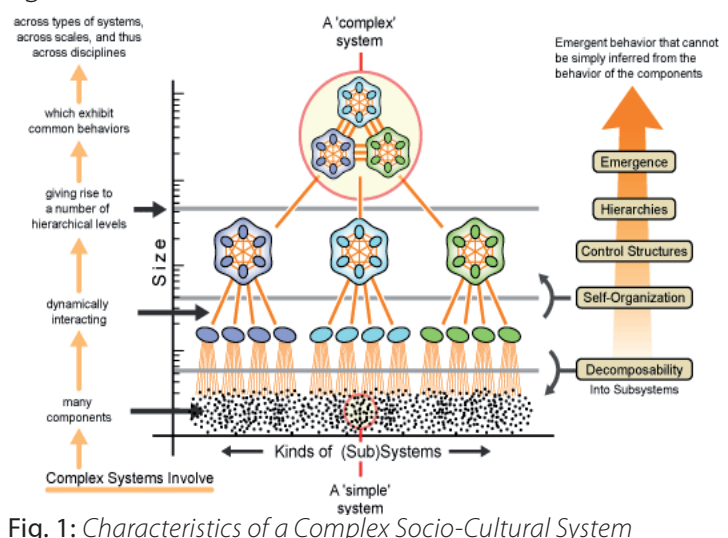

(Modified from original by Abraham et al. 2010).

Complex systems do not exist in isolation - they interact with their environment; they can emerge as the result of sub-systems; as well as form part of other larger complex systems. When considering the interactions of the various social network elements, HSCB researchers noticed the need to investigate the interactions of the emergent systems properties - the higher level features; interdependencies between these elements and emergent properties; the multiple nested levels and hierarchies that can form; and if the elements and properties are included in other systems.

According to D'Andrea (2010) the interest in 
CSNA has been rapidly growing in recent years. Economists, sociologists, software engineers, psychologists and mathematicians have given significant contributions, making it an interdisciplinary research field (D'Andrea, 2010). A comprehensive HSCB development framework will be composed of methods used to rapidly collect and visualize socio-cultural complex network data to analyze interactions between people, groups and organizations by integrating knowledge from available databases and survey results from open source repositories (Karwowski \& Ahram, 2011). According to D'Andrea (2010), the first category of methods are those used for network data collection, these methods aim to provide a dataset that will support studying the effects of social networks on various aspects of social and cognitive activities.

To analyze large datasets, the following methods were suggested summarized by D'Andrea (2010): (a) Socio-centric modeling: to investigate collective relationships for analytical. (b) Egocentric: to allocate main actors, and identify their connected nodes. The second group of methods used for data visualization, these methods aim to represent data in usable and easy to visualize innovative graphical formats, thus making complex information understandable by decision makers and within the military and peace keeping delegates at various levels (policy, combat operations) and to support cultural and social situational awareness for strategic decision-making and training.

- Maps: to manage a wide amount of data and information;

-Matrices: to visualize dense socio-cultural networks;

- Graphs: to visualize relationships among members of a narrow socio-cultural Network;

- Hybrid approach: to integrate different visualization perspectives according to the rapid sociocultural assessment goals.

According to Ahram and Karwowski (2011) and Frias-Martinez et al. (2005) machine learning techniques have limitations for modeling human sociocultural behavior, the main reason is the poor reference to the inherent uncertainty associated with human decision-making process. This limitation can investigate and avoided with the introduction of Soft-computing (SC) for human socio-cultural modeling. According to Frias-Martinez et al. (2005):

"SC is an innovative approach to building computationally intelligent systems that differs from conventional (hard) computing in that it is tolerant of imprecision, uncertainty and partial truth. The guiding principle of soft computing is to exploit the tolerance for imprecision, uncertainty and partial truth to achieve tractability, robustness and low solution cost. SC consists of several computing approaches, including neural networks, fuzzy set theory, approximate reasoning, and search methods, such as genetic and evolutionary algorithms."

According to Ahram and Karwowski (2011): "SC techniques provide an approximate solutions to an ill-defined problems and can help creating human socio-cultural behavioral models in an environment, such as during conflicts, in which users are not willing to give feedback on their actions and/or not able to fully define all possible interactions due to social and cultural barriers".

Different techniques provide different capabilities to support extensive cultural situationalawareness analysis for tactical decision-making and rapid socio-cultural assessment (Ahram, Karwowski and Amaba, 2011). As an example, FriasMartinez et al. (2005) indicated that Fuzzy inference system provides an approach to imitate and simulate decision-making process used to derive goals and plans; Neural Networks provide a flexible technique for representing similar characteristics of individual stereotypes (Frias-Martinez et al., 2005). Generated models based on available datasets can be classified in this work based on human behaviors representation as models, and their purpose or connectivity as shown in Figure 2. According to Ahram and Karwowski (2012a) two main dimensions are considered:

\section{1)Type of task:}

- Filtering,

- Classification,

- Prediction, and

\section{- Recommendation}

2)Granularity of the model, i.e. for each individual (content-based modeling) or for group of users (collaborative modeling).

Prediction can be defined as "the capability of anticipating future actions using past behavior" (Ahram and Karwowski, 2012a). An assumption is made with this approach is that user's short term 
future is very similar to his/her immediate or midterm past; this is commonly presented as contentbased filtering. Recommendation is the capability of suggesting interesting socio-cultural dataset elements relationships; According to Ghanmi et al. (2007) "clustering is the process of locating in- teresting data and groups of interests from among the data. It is a technique that groups data with similar characteristics". The benefit of visualization is to map data onto a graphical representation and to provide a qualitative correlation to various properties.

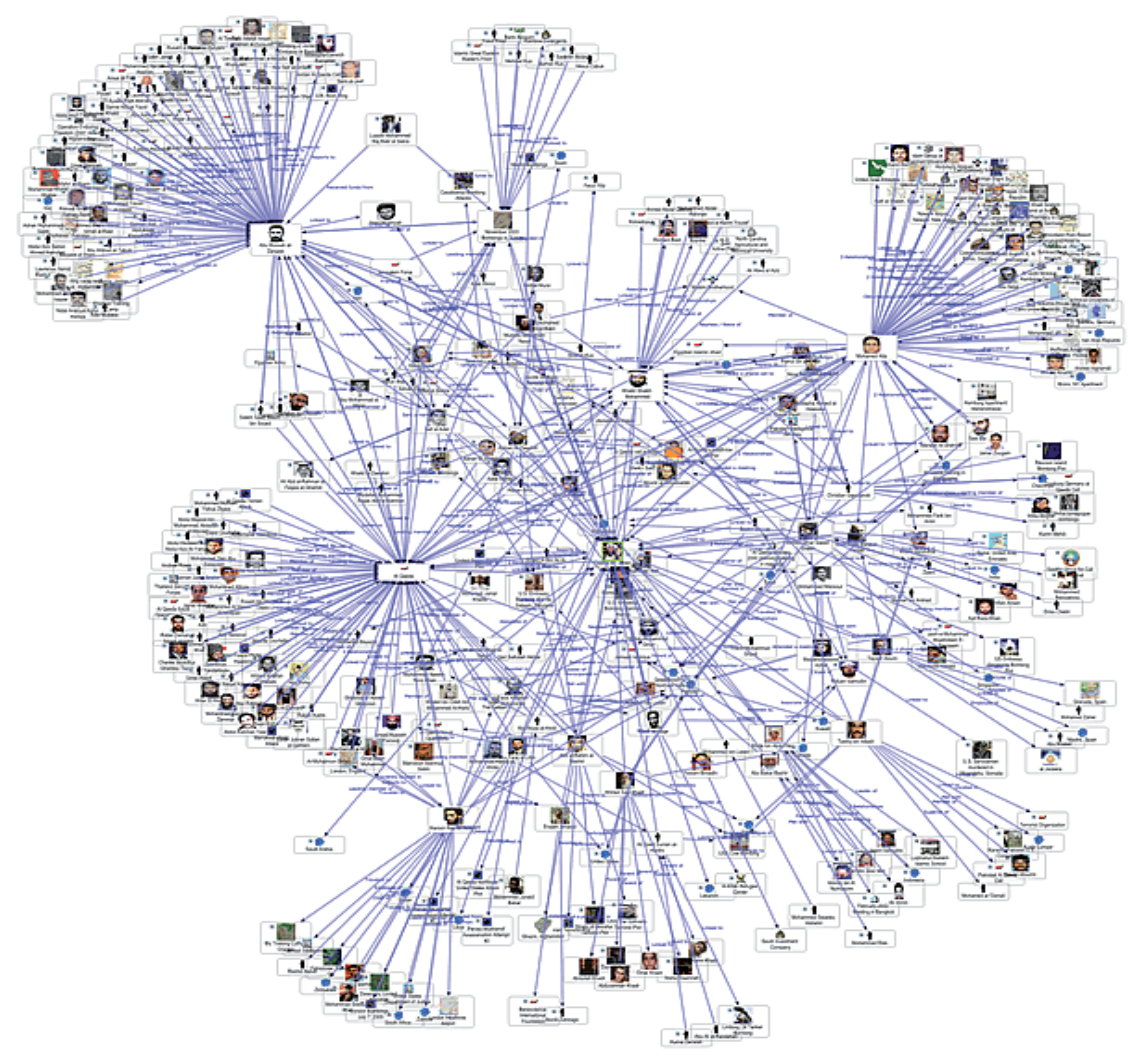

Fig. 2: Example Visual Socio-cultural Network Analysis. (Source: http://lancestrate.blogspot.com).

According to Kohonen 2001), Self-Organizing Map's (SOM) is a neural network model for clustering and visualizing high-dimensional data. The SOM can be utilized to render high-dimensional complex socio-cultural data onto a low-dimensional allocation, usually two dimensional (Kohonen, 2001, Ghanmi et al., 2007). According to D'Andrea (2010), data visualization is used as a way to cluster and categorize large quantities of data and present them to: 2) discover hidden relationships, 1) communicate messages, 3) insight or get perspective insight into aspects already known relationships. The visualization can be performed by using matrices for actors and properties, graphs, maps, or a combined hybrid approach.
One of the key benefits of this HSCB research is the identification of algorithms to automate appropriate labor intensive data analysis and filtering associated with creating an assessment of databases. The following is a list of algorithms identified. Tables 1 summarizes a special class of soft-computing techniques with respect to interpretability and forecasting of events associated with high importance to socio-cultural data analysis. It is imperative to notice that the ability to handle noise in datasets, i.e., how noise activity will affect the models produced. The interpretability of the results, in terms of how easy it is for a military personnel and decision-makers at various levels, policy, combat operations, to understand and interpret the 
knowledge captured from the models generated results, is considered a critical dimension in the rapid ethnographic assessment tool design, as interpretation and forecasting is often cited as a critical problem in most research problems especially data mining methods.

Table 1: Example of selected soft-computing techniques.

\begin{tabular}{|l|l|}
\hline Type of Task & Method \\
\hline Filtering & Diffusion Maps \\
\hline Prediction & Fuzzy Clustering \\
\hline Recommendation & $\begin{array}{l}\text { Self-Organizing Map's, } \\
\text { Cellular automata }\end{array}$ \\
\hline
\end{tabular}

\section{Cellular automata (CA): Example Soft-compu- tational Method Applied to HSCB Modeling}

Cellular automata (CA) is a computational methodology for simulating different sophisticated natural phenomena. CA has many applications in military including but not limited to the areas of cryptology, intrusion detection, modeling cultural change and policy making. CA is composed of a set of computing cells which interact locally and synchronously with each other to determine their next state. CA creates complex patterns at the final state of the system after numerous iterations of small changes on the state of cells. Preliminary experimental analysis performed on a discrete set of spatial data representing factors from social and economic data demonstrated that the use of CA has several significant contributions to support decisions makers allocating development efforts to better stabilize regions associated with higher risks, and to better understand the nonlinearity and complex socio-economic and cultural interactions with adverse events.

According to Thirumaran et al. (2012) a discrete cellular state space $\mathcal{L}$ is "a set of Finite State Automata (FSAs) that is typically created by a regular d-dimensional lattice of FSAs". For example, a two dimensional cellular automaton consists of a lattice of $\mathcal{L}=m \times n$ where each square is called a cell and reflected by one FSA (Hoekstra et al., 2010). A local value space $\sum$ defines possible states for each FSA. Where the state of each FSA can be in one of the finite number of states. The collective states of all cells create what's called a state space of the complete CA as shown in Eq. (1) (Thirumaran et al. 2012).

$\sigma \in \Sigma \equiv\{0,1,2,3,4, \ldots, k-1, k\}$

Thirumaran et al. (2012) indicated that "a neighborhood $N$ is created by a set of $N$ topologicallyneighboring cells which are influencing a change of state of each updated cell in the next simulation step". Figure 3 shows several examples of hexagons, von Neumann, Moore and Margolus neighborhood arrangements (Von Neumann et al. 1966; Codd 1968; Banks 1970; Margolus 1984; Thirumaran et al. 2012). Boundary conditions support fixed, periodic or reflects behaiour among others (Hoekstra et al., 2010). A transition rule $\phi$ describe change of each updated cell from its "current state" value to a "new state", operating over neighborhood with size $N$ defined in Eq. (2)
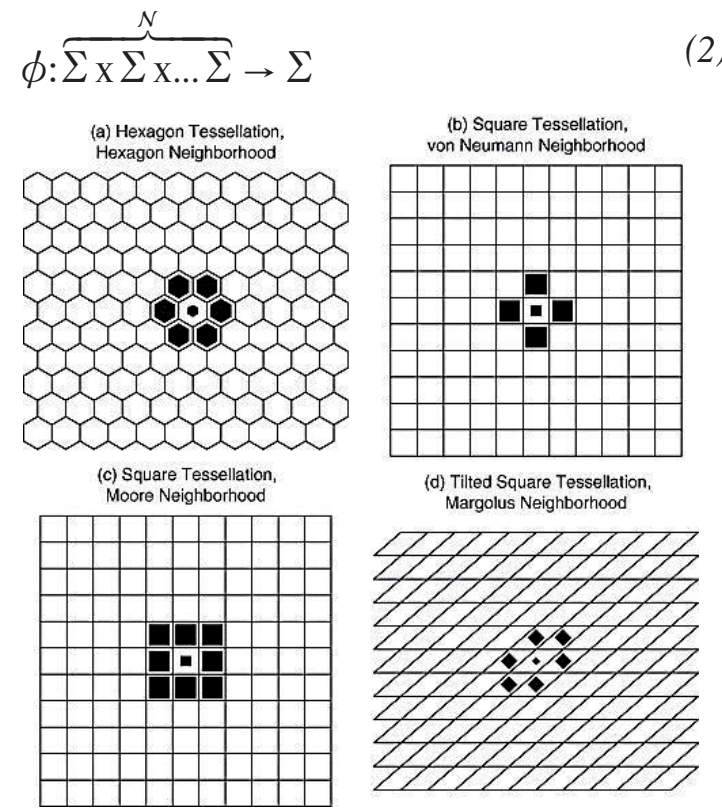

Fig. 3: Various types of neighborhoods (White \& Kiester, 2008)

All cells change their states in a synchronous behaviour at an externally provided clock step. This is called the iteration step (Hoekstra et al., 2010). The definition of von Neumann neighborhood with one unit radius is given below in (Eq.5)

$$
\begin{aligned}
& \mathcal{N}_{m}^{1}(i, j)=\left\{\sigma_{k l l}|i-k|+|j-1| \leq 1\right\}= \\
& \left\{\sigma_{i, j}, \sigma_{j, i-1}, \sigma_{i, j-1}, \sigma_{j, i+1}, \sigma_{i, j+1}\right\}
\end{aligned}
$$


A transition rule $\phi(t)$ can be represented in the form of a function $\phi(t)$ using states $\sigma(t)$ of all cells in the neighborhood (Hoekstra et al., 2010).

$$
\begin{aligned}
& \sigma_{i, j}(t+1)=\phi\left(\sigma_{k, l}(t) \mid \sigma_{k, l}(t) \in \mathcal{N}\right) \\
& \sigma_{i, j}(t+1)= \\
& \phi\left(\sigma_{i, j}(t), \sigma_{j, i-1}(t), \sigma_{i, j-1}(t), \sigma_{j, i+1}(t), \sigma_{i, j+1}(t)\right)
\end{aligned}
$$

CA modeling approach support socio-cultural analysis through a model simulator capable of generating behavioral forecast for events using historical or current data. The CA simulator adapts itself to new datasets to generate forecast based on events or economic factors to use on different locations and with different cultures, this simulator will support generating "what-if" scenarios to forecast adverse events under controlled environments under given time and budget constraints in order to best utilize resources to stabilize and improve various regions. An irregular CA neighborhood map was generated by the researchers using ArcGIS geo-statistical analyst tool. The results of the application of CA HSCB analysis is shown in Figure 4.
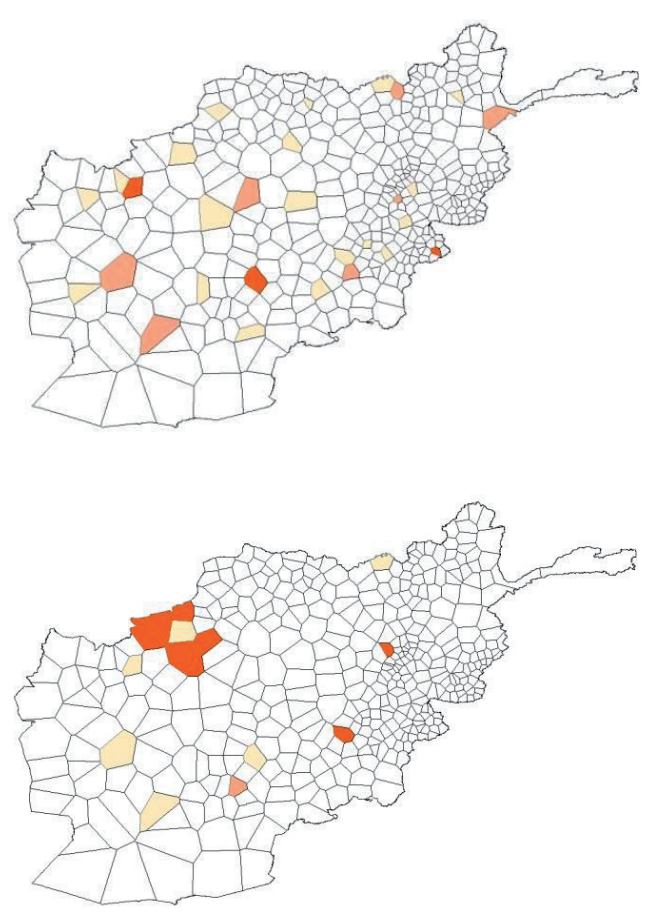

Fig. 4: Example cellular automata voronoi forecasts using irregular grid structure.
In Figure 4, Voronoi diagram was used to visually represent neighborhood $N^{d}$. $\mathcal{N}^{d}$ and defined as a set of any $1^{\text {st }}$ order neighbor that has a common border with the center district $d$. The software platforms used conduct the experimental run and CA research are; ArcGIS, Matlab, Minitab, and Microsoft Access. ArcGIS is used to generate a list of first order neighboring polygons. ArcGIS is also used to determine names and identification numbers of districts using the latitude and longitude information. Microsoft Access used for data processing and structure different test sets. MATLAB was used to process the data and implement CA prediction algorithm, while Minitab used to perform statistical analysis.

\section{Conclusion}

The research investigation of socio-cultural softcomputational modeling examines a unique class of problems relevant to the human social, cultural and behavioral (HSCB) decision making, and investigates the development of a hybrid socio-cultural behavior assessment model and the design the software framework needed to enhance the ability of social and cultural researchers and military decision makers to interpret and explore mission's data in order to forecast and predict anomalous individual and group behaviors. The investigation also supports extensive analysis of cultural situational awareness for strategic decision-making and rapid socio-cultural assessment.

Capturing, encoding and modeling of such phenomena, i.e. uncertainty and variability, became an increasingly important issue in modeling human, cultural and social inter-relationships and integrates both social network and cultural domain analysis. Advances in computational social network analysis (CSNA), social network analysis (SNA) and cultural domain analysis (CDA) will support the effective integration of soft-computing, clustering and dimensionality reduction techniques, such as, but not limited to, those techniques listed in this research. This paper provides an example of soft-computational modeling application using CA, experimental analysis conducted using geostatistical data demonstrates the benefits of this methodology to this class of problems in order to visualize and more accurately interpret the impact of economic factors on regional stability and public safety. This ongoing research address the dilem- 
ma in HSCB-related uncertainty and imprecision in modeling human behaiour by offering a hybrid visual modeling approach that can better assess imprecision and uncertainty inherent to human perceptual and cognitive capacities and support short term forecasting in relation to social, cultural and behavioral factors.

\section{Acknowledgements}

This research was sponsored by the Office of $\mathrm{Na}$ val Research Contract No. N00014-11-1-0934. The authors acknowledge the helpful guidance and support of CAPT Dylan Schmorrow at the Office of Naval Research (ONR) HSCB Program Management. The authors would like to acknowledge the contribution of the HSCB research group and Mr. Halil Bozkurt, PhD Candidate at the University of Central Florida, Institute for Advanced Systems Engineering (IASE).

\section{References and Notes}

[1] Abraham, A., Hassanien, A. E., Snášel, V. (Eds.) (2010). "Computational social network analysis trends, tools and research advances", Dordrecht: Springer, London

[2] Ahram, T. Z., Karwowski, W., (2012a). "Visual Social Network Analysis: Effective Approach to Model Complex Human Social, Behavior \& Culture". The 18th World Congress on Ergonomics (IEA) held February 12-16, 2012, in Recife, Brazil.

[3] Ahram, T. Z., Karwowski, W. (2012b) "Complex Systems Engineering for Rapid Computational Socio-Cultural Network Analysis and Decision Support Systems", Proceedings of the ssecond International Conference on Social Eco-Informatics (SOTICS 2012), Venice, Italy (October 21 26, 2012), (pp 61-67), ISBN: 978-1-61208-228-8.

[4] Ahram, T., Karwowski, W. and Amaba, B.(2011) "Collaborative systems engineering and social networking approach to design and modeling of smarter products", Behav. Inf. Technol. 30, 1 (January 2011), 13-26.

[5] Ahram, T. Z., Karwowski, (2011). "Social Networking Applications: Smarter Product Design for Complex Human Behaviour Modeling", Human Centered Design, Lecture Notes in Computer Science Human Computer Interaction Proceedings. 14th International Conference on HumanComputer Interaction (HCII 2011), Orlando, FL (July 9-14, 2011), Volume 6776, 2011, pp 471-480.

[6] Aslaksen, E.W. (2009). "Designing Complex System", Boca Raton, FL

[7] Banks, Edwin Roger. (1970, 28-30 Oct. 1970). "Universality in cellular automata". Paper presented at IEEE Conference Record of 11th Annual Symposium on the Switching and
Automata Theory.

[8] Carrington, P. J., and Scott, J. (2011), "The SAGE Handbook of Social Network Analysis", London: Sage, chap. 1, pp. 1-8. [856]

[9] Cilliers, P. (1998). "Complexity and postmodernism : understanding complex systems". London ; New York: Routledge.

[10] Codd, E. F. (1968). “Cellular automata”. New York: Academic Press.

[11] D'Andrea, Alessia et al. (2010). "An Overview of Methods for Virtual Social Network Analysis". In Abraham, Ajith et al. Computational Social Network Analysis: Trends, Tools and Research Advances. Springer. p. 8. ISBN 978-1-84882-2283.

[12] Frias-Martinez, E., Magoulas, G., Chen, S., Macredie, R. (2005). "Modeling human behavior in user-adaptive systems: Recent advances using soft computing techniques", Expert Systems with Applications, Volume 29, Issue 2, August 2005, Pages 320-329, ISSN 0957-4174, 10.1016/j. eswa.2005.04.005.

[13] Ghanmi, S., Guedri, M., Bouazizi, M. -L., Bouhaddi N. (2007). "Use of Metamodels in the Multi-Objective Optimization of Mechanical Structures with Uncertainties", International Journal for Computational Methods in Engineering Science and Mechanics, Vol. 8, Iss. 5, 2007

[14] Goldenberg, A., Zheng, A., Fienberg, S., and Airoldi, E. (2009), "A Survey of Statistical Network Models," Foundations and Trends $\times R$ in Machine Learning, 2, 129-233. [857]

[15] Hoekstra, Alfons G.; Kroc, Jiř́; Sloot, Peter M. A. (2010). "Simulating Complex Systems by Cellular Automata, Understanding Complex Systems", Volume 0. ISBN 978-3-64212202-6. Springer-Verlag Berlin Heidelberg, 2010, p. 1

[16] Hunter, D., Krivitsky, P., Schweinberger, M. (2012): Computational Statistical Methods for Social Network Models, Journal of Computational and Graphical Statistics, 21:4, 856-882

[17] IWJOC. Irregular Warfare Joint Operating Concept. 11 September 2007. http://www.fas.org/irp/doddir/dod/iw-joc. pdf.

[18] Karwowski, W., Ahram, T. Z., (2011) "Developing Human Social, Cultural, Behavior (HSCB) Ontologies: Visualizing \& Modeling Complex Human Interactions", Presented at the Office of Secretary of Defense Human Social, Culture Behavior Modeling Conference (HSCB Focus 2011) (February 8-10, 2011). Chantilly, VA

[19] Knoke, D., Yang, S., \& Knoke, D. (2008). “Social network analysis". Los Angeles: Sage Publications.

[20] Kohonen, T. (2001). "Self-Organizing Maps", New York: springer. 
[21] Kolaczyk, E. D. (2009), "Statistical Analysis of Network Data: Methods and Models", New York: Springer. [857]

[22] Margolus, Norman. (1984). "Physics-like models of computation". Physica D: Nonlinear Phenomena, 10(1-2), 81-95. doi: 10.1016/0167-2789(84)90252-5

[23] Miller, J. H., \& Page, S. E. (2007). "Complex adaptive systems: an introduction to computational models of social life", John H. Miller and Scott E. Page. Princeton, New Jersey: Princeton University Press.

[24] Numrich, S.K., and Tolk, A. (2010). "Challenges for Human, Social, Cultural, and Behavioral Modeling". SCS M\&S Magazine 1(1).

[25] Schmorrow, D., Nicholson, D. (Eds.) (2010). "Advances in Cross-Cultural Decision Making, Volume 3 of Advances in Human Factors and Ergonomics Series. Taylor \& Francis, Boca Raton, FL

[26] Thirumaran, M., Dhavachelvan, P., Aishwarya, D., Kiran Kumar Reddy (2012). "Evaluation of Change Factors for Web Service Change Management", Procedia Technology, Volume 6, 2012, Pages 163-170, ISSN 2212-0173, 10.1016/j. protcy.2012.10.020.

[27] Von Neumann, John, Burks, Arthur W., \& Burks, Arthur Walter ed. (1966). "Theory of self-reproducing automata". Edited and completed by Arthur W. Burks. Urbana: University of Illinois Press.

[28] White, Denis, \& Kiester, A. Ross. (2008). "Topology matters: Network topology affects outcomes from community ecology neutral models. Computers", Environment and Urban Systems, 32(2), 165-171. doi: 10.1016/j.compenvurbsys.2007.11.002 